\title{
High 3D:5D ratio: A possible correlate of externalizing and internalizing problems. An exploratory study
}

\author{
Esther I. de Bruin, PhD* \\ Pieter F.A. de Nijs, MD PhD* \\ Anja C. Huizink, PhD $^{\star *, * \star *}$ \\ Frank C. Verhulst, MD PhD*
}

* Department of Child and Adolescent Psychiatry, Erasmus University Medical Centre Rotterdam/Sophia Children's Hospital

** University of Amsterdam, Faculty of

Behavioral and Social Sciences, Research Institute of Child Development and Education

*** Radboud University Nijmegen, Behavioral Science Institute, Nijmegen

THE NETHERLANDS

\begin{abstract}
Background and Objectives: The second to fourth (2D:4D) digit ratio is a sexually dimorphic trait which has been studied to examine the association between fetal hormones and a variety of behaviors. Lower 2D:4D ratios, suggestive of exposure to higher levels of prenatal testosterone, have been associated with male-linked disorders, while higher 2D:4D ratios, suggestive of exposure to weaker prenatal androgen action, have been associated with female-linked disorders. Past research has concentrated on the $2 \mathrm{D}: 4 \mathrm{D}$ ratio, whereas the relationship between other ratios, such as the $3 \mathrm{D}: 5 \mathrm{D}$ ratio, and psychopathology has not much been studied before. Therefore, the aim of this study was to assess the correlation between the 2D:4D and 3D:5D ratio, and internalizing as well as externalizing symptoms, in a large non-clinical sample (143 boys, 150 girls) of white Caucasian children aged 7 to 13 years.

Methods: Externalizing and internalizing symptoms were assessed with the Child Behavior Checklist (CBCL).

Results: The 3D:5D ratio in boys and in girls was positively associated with scores on Externalizing Problems. Further, in girls only, the 3D:5D ratio was positively correlated to scores on Internalizing Problems.

Conclusions: The 3D:5D ratio can be considered a correlate of externalizing and internalizing problems in children from the general population.
\end{abstract}


In humans, the critical period in prenatal life for sexual differentiation starts around the $7^{\text {th }}-8^{\text {th }}$ week after conception ${ }^{1}$. Until this moment the genitalia are undifferentiated and the level of testosterone determines whether the fetus then starts to develop into male or female. Prenatal hormones that influence the sexual differentiation also have an effect on sexual differences in behavior.

Fetal hormones and its effects on behavior can be studied in a variety of ways. Direct measurements such as hormone levels being extracted from the umbilical cord at birth or assessing hormonal levels of the fetus or the mother during pregnancy suffer from disadvantages due to their invasive nature, selection biases, and ethical considerations. Researchers interested in the effect of prenatal hormones on the behavior of children or adults, therefore often rely on indirect measures. Cohen-Bendahan and colleagues ${ }^{2}$ described three indirect methods of examining the behavioral effects of prenatal exposure to sex hormones: otoaucoustic emissions, finger length ratio, and dermatoglyphics. In this paper, we will focus on finger length ratios.

Since $1875^{3}$, it is known that the length of the index finger, compared to the length of the ring finger, the $2 \mathrm{D}: 4 \mathrm{D}$ ratio, is a sexually dimorphic trait, not only in humans ${ }^{4}$ but also in bonobos ${ }^{5}$. Lower ratios are more common in men and ratios closer to one are more characteristic of women ${ }^{6,7}$. The relative length of fingers is fixed for life within the first three months of pregnancy ${ }^{6,8}$. Finger length ratio is considered as a marker of the amount of testosterone the fetus was exposed to in the womb. A higher level of prenatal testosterone is associated with more male-like behaviors, and with a lower 2D: $4 \mathrm{D}$ ratio, and vice versa, a high $2 \mathrm{D}: 4 \mathrm{D}$ ratio is associated with lower levels of fetal testosterone ${ }^{6,9}$. McFadden and Shubel ${ }^{10}$ showed the largest sex differences in 2D:4D, 2D:5D and 3D:4D, but also 3D:5D differed significantly between the sexes. And more recently, it was found that across different studies, the 2D:5D was the most and the 2D:3D was the least sexually differentiated ${ }^{11}$.

Since 1875 many association studies with the 2D:4D ratio have been carried out (currently a 2D:4D digit ratio search in five scientific databases leads to 757 hits, as compared to 22 hits for a 3D:5D digit ratio search). A large number of studies have related the $2 \mathrm{D}: 4 \mathrm{D}$ ratio to a variety of variables (i.e., assertiveness, breast cancer, fertility, hand preference, homosexuality ${ }^{4,12}$. More recently the 2D:4D ratio has been associated with psychiatric traits, personality, and social behaviors that show sex differences $^{13-15}$. However, for other finger length ratios, such as for instance the 3D:5D ratio, hardly any studies are available. Since McIntyre et al. ${ }^{16}$ found that among children, comparison with 3D distinguishes the sexes much better than with 2D and McFadden and Bracht ${ }^{17}$ found that the largest effect sizes were demonstrated in ratios that involved the $5^{\text {th }}$ digit, we considered the $3 \mathrm{D}$ : $5 \mathrm{D}$ ratio a logical choice of ratio besides the more commonly studied 2D:4D ratio. Therefore the topic of this paper is to assess the association between the 3D:5D ratio, and psychopathology, more specifically, internalizing and externalizing symptoms.

With respect to digit ratios, prenatal testosterone, and the association with externalizing behavior, some evidence stems from studies in patients with congenital adrenal hyperplasia $(\mathrm{CAH})$. These individuals were exposed to high levels of testosterone in the womb. Later in life they exhibit more masculine digit ratios and externalizing behavior than normal controls ${ }^{18,19}$. However, results across studies are inconsistent because for instance Buck and colleagues ${ }^{20}$ did not find masculinized digit ratios in patients with $\mathrm{CAH}$. 
Further, Stevenson et al. ${ }^{21}$ found associations between a low 2D:4D ratio in female college students and symptoms of AttentionDeficit/Hyperactivity Disorder (ADHD). Similarly, Martel et al. ${ }^{22}$ showed that a masculinized 2D:4D ratio was correlated with ADHD symptoms in boys. Although they did not study the 3D:5D ratio, the authors assessed the 3D:4D ratio, and found a similar negative association with ADHD symptoms. De Bruin et al. ${ }^{15}$ showed that boys with ADHD or Oppositional Defiant Disorder (ODD) had significantly lower 2D:4D ratios than boys with anxiety disorders, who had a more female-like ratio. Further, a lower 2D:4D ratio was found to be associated with more aggression in men ${ }^{13}$.

As for the 3D:5D ratio, Stevenson et al..$^{21}$ found a weak positive correlation between the right hand $3 \mathrm{D}: 5 \mathrm{D}$ ratio and $\mathrm{ADHD}$ symptoms in males. Further, McFadden et $a l .{ }^{23}$ showed that boys with ADHD actually differed more from control boys on the 3D:5D ratio (and on the 2D:5D, and 4D:5D ratio) than on the more common $2 \mathrm{D}: 4 \mathrm{D}$ ratio which only showed small differences across groups.

With respect to the association of digit ratios and internalizing problems, it was found that men with a higher $2 \mathrm{D}: 4 \mathrm{D}$ ratio reported higher levels of anxiety ${ }^{24}$, and that depression in men was associated with more female-like digit ratios ${ }^{14}$. So far, no studies of the association between anxiety, depression, and the 3D:5D ratio have been found.

Summarized, previous studies have mostly focused on the 2D:4D ratio and it is therefore unknown whether the 2D:4D ratio can be considered a specific indicator for psychiatric traits. Previous studies recommend to study other ratios as well, and a currently no information is available about the possible correlations between the $3 \mathrm{D}: 5 \mathrm{D}$ ratio and psychopathology in the general (child) population. Therefore the aim of this study was to assess the association between internalizing symptoms such as anxiety and depression, externalizing symptoms such as aggression, on the one hand, and the more common 2D:4D ratio, as well as the less explored 3D:5D ratio, on the other, in a large sample of children from the general population. Since the 3D:5D ratio follows the same sexual dimorphic pattern as the 2D:4D ratio, albeit to a lesser extent, we expected to find associations in a similar direction. Thus, we expected a lower 3D:5D ratio (and 2D:4D ratio) to be associated with more externalizing problems, and a higher 3D:5D ratio (and 2D:4D ratio) to be associated with more internalizing problems.

\section{Methods}

\section{Participants}

The sample consisted of 293 white Caucasian children, aged $7-13$ years $(M=$ $10.3, S D=1.5$ ), 143 boys and 150 girls (no siblings) from the general population. Hand scans were collected as part of a bigger study in the general Dutch population (for details see ${ }^{25}$ ). The $\mathrm{CBCL}^{26}$ was sent to parents, and hand scans were made when children visited our department. Out of 293 children, two of the parents did not fill out the CBCL, which yielded 291 children. In $90 \%$ of the cases the CBCL was filled out by the mother, and in $10 \%$ of the cases the father filled out the CBCL. For 27 children no hand scan was made due to logistic or technical difficulties at the time of testing. Further, for 42-47 children (varying per digit ratio), either the $2 \mathrm{D}: 4 \mathrm{D}$ or the $3 \mathrm{D}: 5 \mathrm{D}$ ratio could not be calculated due to one or more of the digits for the left or the right 
hand being insufficiently visible to carry out adequate measurements. This yielded a minimum sample of 217 children for whom at least one measured hand scan and CBCL data were available. The maximum sample consisted of 222 children.

Parents had all signed informed consent forms prior to participation in the study. Children of 12 or 13 years old signed the consent forms themselves as well. The Medical Ethics Committee of the Erasmus Medical Center approved the study.

\section{Assessment}

\section{$C B C L$}

The reliable and valid CBCL was used to obtain standardized parent-reports on children's problem behaviors ${ }^{26}$. Parent(s) or caretaker(s) were asked to rate the occurrence of problems in the preceding six months. The Internalizing Problems broadband scale consists of the sum of scores on the syndrome scales Withdrawn, Somatic Complaints, and Anxious/Depressed. The Externalizing Problems broadband scale consists of the sum of scores on the Delinquent and Aggressive Behavior syndrome scales. T-scores were calculated; T-scores from 60-63 were considered to be in the borderline clinical range, and T-scores of 63 and above were classified as clinical range ${ }^{26}$. For Externalizing Problems, $15.6 \%$ of boys had scores in the borderline clinical or clinical range (7.8\% versus $7.8 \%$ respectively) and for girls this was substantially lower $(3.3 \%$ versus $4.7 \%$ ). With respect to the Internalizing Problems, $26.9 \%$ of the boys had scores in the borderline clinical or clinical range ( $11.3 \%$ versus $15.6 \%$ respectively). For girls, this was $22.7 \%$ ( $10.7 \%$ in the borderline clinical range, and $12.0 \%$ in the clinical range).

\section{Digit ratios}

Children were requested to press their hands flat on a scanner attached to a personal computer. Subsequently, finger length measurements of all digit ratios were made from the basal crease to the tip of the finger with electronic vernier calipers reading to $0.01 \mathrm{~mm}$. Manning et al. ${ }^{6}$ showed that this type of measurement is very reliable.

High inter-rater reliability in our studies has been shown ${ }^{15}$. Three independent raters rated 30 randomly selected pairs of hands. Intra-class correlation coefficients (ICCs) of the left (0.87) and the right hand (0.86) were good. Agreement for the left hand ratio $(k=0.96)$ and the right hand ratio $(k=0.96)$ were excellent. Further, an intra-rater reliability study was carried out. One rater rated the same 30 pairs of hands twice. ICC showed excellent agreement (ICC $=0.95$ for left hand, and ICC $=0.91$ for right hand).

\section{Statistical Analysis}

An independent samples $t$-test was used to test the difference in left and right hand ratios between boys and girls. Means and standard deviations for the left and the right hand 2D:4D and 3D:5D ratios were calculated for boys and girls separately.

Further, due to not-normal distributions of the Externalizing as well as the Internalizing broadband scales (Shapiro-Wilk test: $p<0.01$ ) non-parametric correlations (Spearman's $\rho$ ) were calculated between digit ratios on the one hand, and CBCL Externalizing and Internalizing broadband scale T-scores on the other. 


\section{Results}

\section{Descriptives}

In Table 1 means and standard deviations of the 2D:4D and 3D:5D ratio for boys and girls are presented. Boys and girls showed a significant difference with respect to the left hand 3D:5D ratio $(t[220]=-2.209 ; p=$ $0.03)$. Although no significant sex differences were found in 2D:4D ratio, we choose to run and describe 2D:4D analyses separately per sex as well, to make comparison to $3 \mathrm{D}: 5 \mathrm{D}$ ratio easier.

Table 1

Descriptives for boys and girls for the left and right hand 2D:4D and 3D:5D ratio $(n=104-118)^{*}$

Digit ratios

Boys

Girls

\begin{tabular}{lllll}
\hline & M & SD & M & SD \\
\hline Left hand 2D:4D ratio & 0.9568 & 0.032 & 0.9625 & 0.033 \\
\hline Right hand 2D:4D ratio & 0.9591 & 0.035 & 0.9592 & 0.034 \\
\hline Left hand 3D:5D ratio & 1.296 & 0.049 & 1.312 & 0.057 \\
\hline Right hand 3D:5D ratio & 1.297 & 0.054 & 1.308 & 0.060 \\
\hline
\end{tabular}

Note: $\mathrm{M}=$ Mean; SD = Standard Deviation; $* n$ varies between 104 and 118 for the different ratios.

Table 2

Correlations for boys $(\mathrm{n}=104-105) *$ and girls $(\mathrm{n}=112-118) * *$ between left and right hand digit ratios and T-scores of CBCL Externalizing and Internalizing Problems

$\begin{array}{lll}\text { CBCL broad band scales boys } & \text { LH } & \text { RH }\end{array}$

\begin{tabular}{lcccc}
\hline & $2 \mathrm{D}: 4 \mathrm{D}$ & $3 \mathrm{D}: 5 \mathrm{D}$ & $2 \mathrm{D}: 4 \mathrm{D}$ & 3D:5D \\
\hline Internalizing Problems & $\rho=0.12 ; p=0.22$ & $\rho=0.19 ; p=0.06$ & $\rho=-0.01 ; p=0.90$ & $\rho=0.11 ; p=0.26$ \\
Externalizing Problems & $\rho=-0.08 ; p=0.41$ & $\rho=0.21 ; p=0.04$ & $\rho=-0.16 ; p=0.11$ & $\rho=-0.02 ; p=0.88$
\end{tabular}

CBCL broad band scales girls

Internalizing Problems $\quad \rho=-0.02 ; p=0.81 \quad \rho=0.20 ; p=0.03 \quad \rho=0.09 ; p=0.37 \quad \rho=0.19 ; p=0.04$

Externalizing Problems $\quad \rho=-0.01 ; p=0.91 \quad \rho=0.21 ; p=0.02 \quad \rho=0.01 ; p=0.91 \quad \rho=0.25 ; p=0.01$

Note: $\mathrm{CBCL}=$ Child Behavior Checklist; $\mathrm{LH}=$ left hand; $\mathrm{RH}=$ right hand. $* n$ varies from 104-105 for the different digit ratios. $* * n$ varies between 112 and 118 for the different digit ratios.

\section{Correlations}

Correlations between CBCL scores and digit ratios are presented in Table 2. In boys, contrary to our hypothesis, the CBCL broadband scale Externalizing Problems showed a positive correlation with the $3 \mathrm{D}: 5 \mathrm{D}$ ratio of the left hand $(\rho=0.21 ; p<0.05)$.

In girls the 3D:5D digit ratio of the left hand showed a positive association with the CBCL broadband scales Externalizing and 
Internalizing Problems $(\rho=0.21 ; p<0.05$ and $\rho=0.20 ; p<0.05$ respectively). The same associations applied to the 3D:5D ratio of the right hand $(\rho=0.25 ; p=0.01$ for Externalizing Problems and $\rho=0.19 ; p$ $<0.05$ for Internalizing Problems). This is partly in accordance with our hypotheses.

\section{Discussion}

This exploratory study described the relationship between the less explored 3D:5D ratio as well as the more common 2D:4D ratio, and psychiatric symptoms. This was studied in a relatively large sample of children from the general population, and externalizing as well as internalizing problems were assessed, whereas in previous studies, samples were usually smaller, and mostly only one symptom dimension was covered.

No significant differences were found between boys and girls for the 2D:4D ratio but we did find a larger 3D:5D ratio for girls as compared to boys which is the sexual dimorphic pattern we expected to find. However, most previous studies showed that the 2D:4D ratio in boys is smaller than in girls and at this point, we have no satisfactory explanation why this study did not replicate these sex differences.

In boys the 3D:5D ratio was positively associated with externalizing problems. A higher 3D:5D ratio was associated with higher scores, as reported by parents, on for instance aggressive behaviour. In some previous studies a negative association was found between the 2D:4D ratio and aggression in men (i.e., ${ }^{13}$ ), but the (opposite) association between the 3D:5D ratio and aggression has not been shown before, and was contrary to our expectations. Since the
3D:5D ratio is also sexually dimorphic, we expected a similar pattern as is found with respect to the $2 \mathrm{D}: 4 \mathrm{D}$ ratio, thus a negative association with externalizing (male-oriented) behavior. From our findings it seems that exposure to high levels of testosterone in the womb as reflected later in life by a high level of male-oriented behavior, is being represented by a high 3D:5D as a biomarker. The 3D:5D would then act as an opposite biomarker to the 2D:4D. Or one could also imagine other hormones besides testosterone, or genetic influences, are at play at the formation of the $3^{\text {rd }}$ and the $5^{\text {th }}$ digit. However, this is currently no more than a preliminary finding and careful interpretation should be made.

For girls, positive associations between the $3 \mathrm{D}: 5 \mathrm{D}$ ratio and externalizing as well as internalizing problems were found for the left and the right hand. A higher 3D:5D ratio was associated with higher scores on items that represent aggressive, delinquent, depressed, anxious and withdrawn behavior. The 3D:5D ratio seemed to be a correlate of overall psychopathology in girls. We would have expected to find this association only for the more internalizing (female-oriented) behaviors, and we expected the more malelinked externalizing behaviors to be negatively correlated to the $3 \mathrm{D}: 5 \mathrm{D}$ ratio, similar to what has previously been demonstrated with respect to the $2 \mathrm{D}: 4 \mathrm{D}$ ratio. Thus, in boys and in girls, the 3D:5D seems so follow an opposite pattern of association (higher 3D:5D is correlated to higher scores on externalizing behavior) to what is found in most 2D:4D ratio studies (higher 2D:4D ratio is correlated to lower scores on externalizing behavior). To be able to provide a satisfactory explanation for these findings, we need to examine the role of other hormones besides testosterone, as well as the role of the (Homeobox) genes in the forma- 
tion of the $3^{\text {rd }}$ and $5^{\text {th }}$ digit, which is beyond the scope of this preliminary study. From the vast amount of previous studies there seems to be sufficient evidence that the 2D:4D ratio can be considered a negative correlate of prenatal testosterone (i.e., ${ }^{7}$ ). We are not aware of any publications of direct measurements of prenatal hormones (via umbilical cord, or fetus' blood) and the development of 3D and 5D. We cannot draw any conclusions about whether and how the 3D:5D ratio is related to prenatal testosterone from this study.

Further, positive correlations between the 2D:4D and internalizing problems as have been demonstrated before ${ }^{14,24}$ were not replicated in this study. We did find that in our sample, relatively high scores were obtained on the Internalizing Broadband scale. About a quarter of the children had scores in the borderline clinical or even clinical range. So, although our sample was randomly drawn from the general population, these fairly high scores are not representative of internalizing problems in general. However, we are not convinced that this would explain why the positive association with the 2D:4D was not replicated.

Besides 2D:4D and 3D:5D ratio, there obviously are other important ratios. For instance, McFadden et al. ${ }^{23}$ showed that the 2D:5D, 3D:5D and 4D:5D ratio revealed much bigger group differences (children with ADHD vs. normal controls) than the more commonly investigated 2D:4D ratio, and they therefore emphasized not only to focus on the 2D:4D ratio. Also Berenbaum et al $^{27}$ state that the interpretation of the 2D:4D ratio as an indicator of prenatal androgen exposure is controversial, with limited direct evidence.

In our sample, we did also assess the associations between all other finger length ratios and externalizing and internalizing pro- blems. It would be beyond the scope of this paper to discuss them all but the main findings, besides all the 3D:5D associations, were that the $4 \mathrm{D}: 5 \mathrm{D}$ ratio roughly showed a similar, opposite to what would be expected, pattern to the 3D:5D ratio (a higher $4 \mathrm{D}: 5 \mathrm{D}$ ratio was related to more externalizing problems) and the 2D:5D ratio showed a positive association with internalizing problems in girls, which is in line with previous 2D:4D findings, and with our 3D:5D results.

To conclude, this exploratory study aimed to broaden the perspective of research into digit ratios by also assessing other finger length ratios, such as the $3 \mathrm{D}: 5 \mathrm{D}$, as possible biomarkers of prenatal processes.

\section{Acknowledgments}

This study was supported financially by a grant from the Netherlands Organization for Scientific Research (NWO/ZonMw/OOG100-002-006).

\section{References}

1. Breedlove SM. Sexual differentiation of the brain and behavior. In: Becker JB, Breedlove SM, Crews D, eds. Behavioral Endocrinology. Cambridge: The MIT Press; 1993. p. 39-71.

2. Cohen-Bendahan CCC, van de Beek C, Berenbaum SA. Prenatal sex hormone effects on child and adult sextyped behavior: methods and findings. Neurosci Biobehav Rev 2005; 29: 353-384.

3. Ecker A. Einige bemerkungen über einen schwankengen charakter in der hand des menschen. Arch Anthropol (Braunschweig) 1875; 8: 67-75.

4. Manning JT, Bundred P. The ratio of $2^{\text {nd }}$ to $4^{\text {th }}$ digit length: A new predictor of disease predisposition? Med Hypotheses 2000; 54: 855-857.

5. McIntyre MH, Herrmann E, Wobber V, Halbwax M, Mohamba C, de Sousa N, et al. Bonobos have a more human-like second-to-fourth finger length ratio (2D:4D) 
than chimpanzees: a hypothesized indication of lower prenatal androgens. J Hum Evol 2009; 54 (4): 361-365.

6. Manning J, Scutt D, Wilson J, Lewis-Jones D. The ratio of 2 nd to 4 th digit length: a predictor of sperm numbers and concentrations of testosterone, luteinizing hormone and oestrogen. Hum Reprod 1998; 13: 3000-3004.

7. Manning JT. Digit ratio: A pointer to fertility, behavior, and health. New Brunswick, NJ: Rutgers University Press; 2002.

8. Garn S, Burdi A, Babler W, Stinson S. Early prenatal attainment of adult metacarpal-phalangeal rankings and proportions. Am J Phys Anthropol 1975; 43: 327-332.

9. Lutchmaya S, Baron-Cohen S, Raggatt P, Knickmeyer R, Manning, JT. $2^{\text {nd }}$ to $4^{\text {th }}$ digit ratios, fetal testosterone and estradiol. Early Hum Dev 2004; 77: 23-28.

10. McFadden D, Schubel E. Relative lengths of fingers and toes in human males and females. Horm Behav 2002; 42: 492-500.

11. Voracek M. Comparative study of digit ratios (2D:4D and other) and novel measures of relative finger length: testing magnitude and consistency of sex differences across samples. Percept Mot Skills 2009;108:83-93.

12. Williams TJ, Pepitone ME, Christensen SE, Cooke BM, Huberman AD, Breedlove NJ, et al. Finger length patterns indicate an influence of fetal androgens on human sexual orientation. Nature 2000; 404: 455-456.

13. Bailey AA, Hurd PL. Finger length ratio (2D:4D) correlates with physical agression in men but not in women. Biol Psychol 2005; 68: 215-222.

14. Bailey AA, Hurd PL. Depression in men is associated with more feminine finger length ratios. Pers Individ Diff 2005; 39: 829-836.

15. De Bruin EI, Verheij F, Wiegman T, Ferdinand RF. Finger length ratio (2D:4D) differences between boys with autism, PDD-NOS, ADHD, and anxiety disorders. Dev Med Child Neurol 2006; 28: 962-965.

16. McIntyre MH, Cohn BA, Ellison PT. Sex dimorphism in digital formulae in children. Am J Phys Anthropol 2006; 129: 143-150.

17. McFadden D, Bracht MS. Sex and race differences in the relative lengths of metacarpals and metatarsals in human skeletons. Early Hum Dev 2009; 85: 117-124.

18. Brown WM, Hines M, Fane BA, Breedlove SM. Masculinized finger length patterns in human males and females with congenital adrenal hyperplasia. Horm Behav 2002; 42: 380-386.
19. Okten A, Kalyoncu M, Yaris N. The ratio of secondand fourth-digit lengths and congenital adrenal hyperplasia due to 21-hydroxylase deficiency. Early Hum Dev 2002; 70: 47-54.

20. Buck JJ, Williams RM, Hughes IA, Acerini CL. In utero exposure and $2^{\text {nd }}$ to $4^{\text {th }}$ digit length ratio-comparisons between healthy controls and females with classical congenital adrenal hyperplasia. Hum Reprod 2003; 18: 967-979.

21. Stevenson JC, Everson PM, Williams DC, Hipskind G, Grimes M, Mahoney ER. Attention deficit/hyperactivity disorder (ADHD) symptoms and digit ratios in a college sample. Am J Hum Biol 2006; 19: 41-50.

22. Martel MM, Gobrogge KL, Breedlove SM, Nigg JT. Masculinized finger length ratios of boys, but not girls, are associated with attention-deficit/hyperactivity disorder. Behav Neurosci 2008; 122: 273-281.

23. McFadden D, Westhafer JG, Pasanen EG, Carlson CL, Tucker DM. Physiological evidence of hypermasculinization in boys with the inattentive type of attentiondeficit/hyperactivity disorder (ADHD). Clin Neurosci Res 2005; 5: 233-245.

24. Evardone M, Alexander GM. Anxiety, sex-linked behaviors, and digit ratios (2D:4D). Arch Sex Behav 2009; 38: 442-455.

25. Tick NT, van der Ende J, Verhulst FC. Twenty-years trends in emotional and behavioral problems in Dutch children in a changing society. Acta Psychiatr Scand 2007; 116: 473-482.

26. Achenbach TM, Rescorla L. Manual for the ASEBA school-age forms \& profiles. Burlington: University of Vermont Research Center for Children, Youth, \& Families; 2001.

27. Berenbaum SA, Bryk KK, Nowak N, Quigley CA, Moffat S. Fingers as a marker of prenatal androgen exposure. Endocrinol 2009; 150: 5119-5124.

Address for correspondence:

Esther I. de Bruin, PhD

Erasmus University Medical Centre Rotterdam/

Sophia Children's Hospital

Dr. Molewaterplein 60

3015 GJ Rotterdam

The Netherlands

Tel. int/31/20/5251262

Fax int/31/20/5251200

E-mail: e.i.debruin@uva.nl 